CERN-PH-TH/2004-163

NRCPS-HE-2004-18

\title{
Tensionless Strings Vertex Operator for Fixed Helicity States
}

\author{
G. Savvidy ${ }^{1}$ \\ CERN Theory Division, CH-1211 Geneva 23, Switzerland \\ Demokritos National Research Center,GR-15310 Athens, Greece
}

\begin{abstract}
The tensionless string theory with perimeter action has pure massless spectrum of higherspin gauge fields. The multiplicity of these massless states grows linearly. It is therefore much less compared with the standard string theory and is larger compared with the field theory models of the Yang-Mills type. It is important to define nontrivial interaction between infinite amount of massless particles of the perimeter string theory. The appropriate vertex operators were defined recently and I study the lowest order vertex operators and the corresponding scattering amplitudes in tree approximation. I emphasize the special importance of the vertex operator for fixed helicity states.
\end{abstract}

\footnotetext{
${ }^{1}$ E-mail: georgios.savvidis@cern.ch
} 


\section{$1 \quad$ Introduction}

A string model which is based on the concept of the surface perimeter ${ }^{2}$ was suggested in $[1,2,3]$. At the classical level the model is tensionless [3] and has a pure massless spectrum of infinitely many integer spin fields $[1,2]$. It was demonstrated in $[2,4]$ that unphysical states are eliminated by the Virasoro and additional Abelian constraints appearing in the model. The analysis of the first three lower levels of the physical Fock space $\mathcal{F}$ shows that the fixed helicity states $\mathcal{F}_{0}$ and the first two excited states $\mathcal{F}_{1,2}$ are well defined and have no negative norm waves. The level $\mathcal{F}_{0}$ of fixed helicity states is infinitely degenerate and contains massless particles of increasing tensor structure $A^{\mu_{1}, \ldots, \mu_{s}}(k)$, where $s=1,2, \ldots$, while the first and the second levels $\mathcal{F}_{1,2}$ are physical null states ${ }^{3}$. It was conjectured in [2] that all excited states $\mathcal{F}_{n} \quad(n \neq 0)$ represent physical null states realizing continuous spin representations of the Poincaré group and define large gauge transformation of the fixed helicity states $\mathcal{F}_{0}$.

The next important issue is connected with the interaction of these massless fields. The above analysis of the physical Fock space imposes strong restriction on the possible form of interactions. Indeed only the subspace $\mathcal{F}_{0}$ represents the propagating physical fields, therefore while introducing interaction into the perimeter string model it is important to demonstrate that nontrivial transitions amplitudes are only between fixed helicity states $\mathcal{F}_{0}$.

The solution of this problem may shed some new light to the old problem of the existence of consistent interaction of massless particles of higher spin fields in flat spacetime $[16,17,18,19]$. The recent attempt to construct the appropriate vertex operators for the perimeter string model was made in [6].

The general form of the suggested vertex operators is given by the formula [6]:

$$
U_{k, \pi}^{\mu_{1} \tilde{\mu}_{1} \ldots, \ldots \mu_{j} \tilde{\mu}_{j}}(\zeta)=: \partial_{\zeta}^{m_{1}} X^{\mu_{1}} \partial_{\bar{\zeta}}^{\tilde{m}_{1}} X^{\tilde{\mu}_{1}} \ldots \quad \ldots \partial_{\zeta}^{m_{j}} \Pi^{\mu_{j}} \partial_{\bar{\zeta}}^{\tilde{m}_{j}} \Pi^{\tilde{\mu}_{j}} e^{i k \cdot X(\zeta)+i \pi \cdot \Pi(\zeta)} \quad:
$$

where the canonically conjugate operators are [1] : $X^{\mu}=\frac{1}{2}\left(X_{L}^{\mu}\left(\zeta^{+}\right)+X_{R}^{\mu}\left(\zeta^{-}\right)\right)$

$$
\begin{aligned}
& X_{L}^{\mu}=x^{\mu}+\pi^{\mu} \zeta^{+}+i \sum_{n \neq 0} \frac{1}{n} \beta_{n}^{\mu} e^{-i n \zeta^{+}}, \\
& X_{R}^{\mu}=x^{\mu}+\pi^{\mu} \zeta^{-}+i \sum_{n \neq 0} \frac{1}{n} \tilde{\beta}_{n}^{\mu} e^{-i n \zeta^{-}},
\end{aligned}
$$

and $P_{\tau}^{\mu}=\partial_{\tau} \Pi^{\mu}$, where $\Pi^{\mu}=\frac{1}{2}\left(\Pi_{L}^{\mu}\left(\zeta^{+}\right)+\Pi_{R}^{\mu}\left(\zeta^{-}\right)\right)$

$$
\begin{aligned}
& \Pi_{L}^{\mu}=e^{\mu}+k^{\mu} \zeta^{+}+i \sum_{n \neq 0} \frac{1}{n} \alpha_{n}^{\mu} e^{-i n \zeta^{+}}, \\
& \Pi_{R}^{\mu}=e^{\mu}+k^{\mu} \zeta^{-}+i \sum_{n \neq 0} \frac{1}{n} \tilde{\alpha}_{n}^{\mu} e^{-i n \zeta^{-}} .
\end{aligned}
$$

The basic commutator is of the form $\left[X_{L, R}^{\mu}\left(\zeta^{ \pm}\right), \partial_{ \pm} \Pi_{L, R}^{\nu}\left(\zeta^{\prime \pm}\right)\right]=2 \pi i \eta^{\mu \nu} \delta\left(\zeta^{ \pm}-\zeta^{\prime \pm}\right)$ and the following commutator relations hold for the oscillators $\alpha_{n}, \beta_{n}$

$$
\left[e^{\mu}, \pi^{\nu}\right]=\left[x^{\mu}, k^{\nu}\right]=i \eta^{\mu \nu}, \quad\left[\alpha_{n}^{\mu}, \beta_{l}^{\nu}\right]=n \eta^{\mu \nu} \delta_{n+l, 0},
$$

\footnotetext{
${ }^{2}$ For the perimeter action $S_{P}=(m / 4 \pi) \int d \tau d \sigma \sqrt{\left(\partial^{2} X\right)^{2}}$ we shall use physical units in which $\mathrm{m}=1$.

${ }^{3}$ In Appendix I discuss the origin of this phenomena at the classical level.
} 
where $\alpha_{0}^{\mu} \equiv k^{\mu}, \quad \beta_{0}^{\mu} \equiv \pi^{\mu}$ is a pair of momentum operators. The momentum $k_{\mu}$ is the standard space-time momentum operator, the $\pi_{\mu}$ is a new momentum operator conjugate to the polarization vector coordinate $e_{\mu} \quad[1]$.

Using the world-sheet energy momentum operator $[1] T(\zeta)=-: \partial_{\zeta} X \cdot \partial_{\zeta} \Pi$ : one can compute the anomalous dimension of the vertex operators in (1) [6]:

$$
\Delta=2(k \cdot \pi)+m_{1}+\tilde{m}_{1}+\ldots \ldots+m_{j}+\tilde{m}_{j}
$$

It must be equal to 2 in order to describe emission of physical states, therefore the corresponding poles are at the points

$$
(k \cdot \pi)=1-\left(m_{1}+\tilde{m}_{1}+\ldots \ldots+m_{j}+\tilde{m}_{j}\right) / 2,
$$

(the conformal spin should be equal to zero, therefore $m_{1}+\ldots+m_{j}=\tilde{m}_{1}+\ldots+\tilde{m}_{j}$ ).

The two lowest order vertex operators of conformal dimension two are the $V_{k, \pi}$ operator:

$$
V_{k, \pi}(\zeta)=: e^{i k \cdot X(\zeta)+i \pi \cdot \Pi(\zeta)}: \quad(k \cdot \pi)=1
$$

and the $U_{k, \pi}$ operators of the type $m=\tilde{m}=1$ :

$$
U_{k, \pi}^{\mu \nu}(\zeta)=: \partial_{\zeta} X^{\mu} \partial_{\bar{\zeta}} \Pi^{\nu} \quad e^{i k \cdot X(\zeta)+i \pi \cdot \Pi(\zeta)}: \quad(k \cdot \pi)=0 .
$$

The $U_{k, \pi}$ operators are of the essential importance, because for them $(k \cdot \pi)=0$ and they create fixed helicity states $\mathcal{F}_{0}[2]$. Indeed the square of the Pauli-Lubanski vector of the Poincaré group is $W=(k \cdot \pi)^{2}=\Xi^{2}$ and defines fixed helicity states, when $W=0$ and continuous spin representations-CSR, when $W \neq 0[7,8,1,2]$.

The aim of this article is to compute the residues of different poles in the scattering amplitudes defined by the above vertex operators and to derive consistency conditions under which all transition amplitudes to the CSR states from fixed helicity states are vanishing. In the next sections I shall present necessary material from [6] and shall derive the consistency conditions under which the residue of the transition amplitude from fixed helicity state $U_{k, \pi}(0) \mid 0>$ to the CSR state $V_{k, \pi}(0) \mid 0>$ is equal to zero.

\section{The Lowest Order Vertex Operators}

Let us consider the lowest order vertex operators in (1), which have been defined as $V_{k, \pi}$ and $U_{k, \pi}$ operators in [6]. The most lower order vertex operator $V_{k, \pi}$ has the form:

$$
V_{k, \pi}(\zeta)=: e^{i k \cdot X(\zeta)+i \pi \cdot \Pi(\zeta)}: \quad(k \cdot \pi)=1
$$

and at the next level there are three relevant operators with $m=\tilde{m}=1[6]$ :

$$
\begin{aligned}
& U_{X X}^{\mu \nu}(k, \pi, \zeta)=:\left(\partial_{\zeta} X^{\mu} \partial_{\bar{\zeta}} X^{\nu}+\partial_{\bar{\zeta}} X^{\mu} \partial_{\zeta} X^{\nu}\right) e^{i k \cdot X(\zeta)+i \pi \cdot \Pi(\zeta)}: \\
& U_{X \Pi}^{\mu \nu}(k, \pi, \zeta)=:\left(\partial_{\zeta} X^{\mu} \partial_{\bar{\zeta}} \Pi^{\nu}+\partial_{\bar{\zeta}} X^{\mu} \partial_{\zeta} \Pi^{\nu}\right) e^{i k \cdot X(\zeta)+i \pi \cdot \Pi(\zeta)}: \quad(k \cdot \pi)=0 \\
& U_{\Pi \Pi}^{\mu \nu}(k, \pi, \zeta)=:\left(\partial_{\zeta} \Pi^{\mu} \partial_{\bar{\zeta}} \Pi^{\nu}+\partial_{\bar{\zeta}} \Pi^{\mu} \partial_{\zeta} \Pi^{\nu}\right) e^{i k \cdot X(\zeta)+i \pi \cdot \Pi(\zeta)}:
\end{aligned}
$$

so that the general form of the fixed helicity state vertex operator $U_{k, \pi}$ is given by the linear combination of these operators

$$
U_{k, \pi}=\omega_{\mu \nu} U_{X X}^{\mu \nu}+2 \varphi_{\mu \nu} U_{X \Pi}^{\mu \nu}+\chi_{\mu \nu} U_{\Pi \Pi}^{\mu \nu},
$$


where $\omega(k, \pi), \varphi(k, \pi)$ and $\chi(k, \pi)$ are polarization tensors, or in our case it will be more precise to call them coupling constants.

There are two types of constraints which are imposed on the physical states and transition amplitudes of the perimeter model: these are the standard Virasoro constraints and the additional Abelian constraints [1]. The general vertex operators (1) are already built in a way to fulfill the conformal invariance of the amplitudes (5). All correlation functions should also be restricted to fulfill the Abelian $\Theta=\Pi^{2}-1=0$ constraint. As a result some of the vertex operators (1) are excluded as unphysical. This happens with the lowest order vertex operator $V_{k, \pi}[6]$. For the vertex operator $V_{k, \pi}$ the Abelian constraint takes the form:

$$
<: \Pi^{2}(\eta):: e^{i k \cdot X(\zeta)+i \pi \cdot \Pi(\zeta)}:>=<: e^{i k \cdot X(\zeta)+i \pi \cdot \Pi(\zeta)}:>.
$$

and reduces to the equation

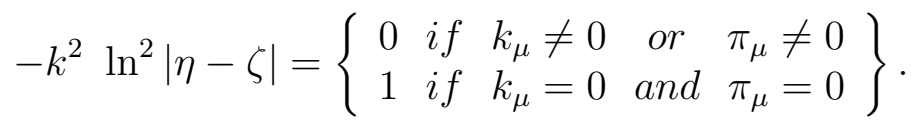

To eliminate the world-sheet coordinate dependence on the l.h.s. we have to impose at least the massless condition

$$
k^{2}=0,
$$

which includes the point $k_{\mu}=0$. Therefore the constraint is fulfilled for nonzero $k_{\mu}$ and breaks down at the point $k_{\mu}=0$. Thus the constraint (9) can not be fulfilled in the deep infrared region where $k_{\mu}=0$ and the vertex operator $V_{k, \pi}$ should be abandoned as unphysical.

The Abelian constraint $\Theta$ should be imposed on the higher order vertex operators $U_{k, \pi}$ as well $<: \Pi^{2}(\eta): U_{k, \pi}(\zeta)>=\left\langle U_{k, \pi}(\zeta)>\right.$ and this condition is fulfilled if $k^{2}=0$ and

$$
\operatorname{Tr} \omega=0 .
$$

Notice that now the V.E.V. on the r.h.s. is equals to zero $\left\langle U_{k, \pi}(\zeta)>=0\right.$.

Because the states created by the operator $V_{k, \pi}$ are unphysical the consistency of the model requires that the transition amplitudes from fixed helicity state $U_{k, \pi}(0) \mid 0>$ to the CSR state $V_{k, \pi}(0) \mid 0>$ must be equal to zero.

Let us therefore consider the vertex operators $U_{k, \pi}$ in more details. These operators have the following operator product expansions [6] with the stress tensor $T(\eta)=-$ : $\partial_{\eta} X \cdot \partial_{\eta} \Pi[1]:$

$$
\begin{aligned}
T(\eta) U_{X X}^{\mu \nu}(\zeta)= & -\frac{1}{(\eta-\zeta)^{3}}:\left(i \pi^{\mu} \partial_{\bar{\zeta}} X^{\nu}+i \pi^{\nu} \partial_{\bar{\zeta}} X^{\mu}\right) e^{i k \cdot X+i \pi \cdot \Pi}: \\
& +\left(\frac{k \pi+1}{(\eta-\zeta)^{2}}+\frac{1}{(\eta-\zeta)} \partial_{\zeta}\right) U_{X X}^{\mu \nu}(\zeta), \\
T(\eta) U_{X \Pi}^{\mu \nu}(\zeta)= & -\frac{1}{(\eta-\zeta)^{3}}:\left(i \pi^{\mu} \partial_{\bar{\zeta}} \Pi^{\nu}+i k^{\nu} \partial_{\bar{\zeta}} X^{\mu}\right) e^{i k \cdot X+i \pi \cdot \Pi:} \\
& +\left(\frac{k \pi+1}{(\eta-\zeta)^{2}}+\frac{1}{(\eta-\zeta)} \partial_{\zeta}\right) U_{X \Pi}^{\mu \nu}(\zeta), \\
T(\eta) U_{\Pi \Pi}^{\mu \nu}(\zeta)= & -\frac{1}{(\eta-\zeta)^{3}}:\left(i k^{\mu} \partial_{\bar{\zeta}} \Pi^{\nu}+i k^{\nu} \partial_{\bar{\zeta}} \Pi^{\mu}\right) e^{i k \cdot X+i \pi \cdot \Pi:} \\
& +\left(\frac{k \pi+1}{(\eta-\zeta)^{2}}+\frac{1}{(\eta-\zeta)} \partial_{\zeta}\right) U_{\Pi \Pi}^{\mu \nu}(\zeta) .
\end{aligned}
$$


The linear combination $U_{k, \pi}=\omega U_{X X}+2 \varphi U_{X \Pi}+\chi U_{\Pi \Pi}$ should be such that the leading singularities $(\eta-\zeta)^{-3}$ cancel, making the operator product characteristic to the conformal dimension-one primary fields. This leads to the equations

$$
\pi^{\mu} \omega_{\mu \nu}+\varphi_{\nu \mu} k^{\mu}=0, \quad \pi^{\mu} \varphi_{\mu \nu}+k^{\mu} \chi_{\mu \nu}=0, \quad(k \cdot \pi)=0 .
$$

An analogous expansion holds between $\bar{T}(\bar{\zeta})$ and the three vertices, leading to the equations

$$
\omega_{\mu \nu} \pi^{\nu}+\varphi_{\mu \nu} k^{\nu}=0, \quad \pi^{\mu} \varphi_{\mu \nu}+\chi_{\nu \mu} k^{\mu}=0, \quad(k \cdot \pi)=0 .
$$

Because the tensors $\omega$ and $\chi$ are symmetric, both equations simply coincide.

The equations (11),(12) and (13) are not the only ones which are imposed on the tensors $\omega, \varphi$ and $\chi$. Indeed, as we just explained the lowest order vertex operator $V_{k, \pi}$ is unphysical and the quantum mechanical consistency requires that the scattering of two states created by the operator $U_{k, \pi}$ into the state created by $V_{k, \pi}$ must vanish. In the next section we shall derive this condition.

\section{The zero residue condition}

Suppose that we are scattering $n$ states which are created by the operators $U_{k, \pi}$. To find the poles and the corresponding residues of the intermediate states, which appear in the tree diagram, we have to compute the operator product of the form

$$
\begin{aligned}
& U_{k_{1}, \pi_{1}}\left(\zeta_{1}\right) U_{k_{2}, \pi_{2}}\left(\zeta_{2}\right) \simeq \mathcal{R} \mathcal{E} \mathcal{S}_{0}\left|\zeta_{1}-\zeta_{2}\right|^{2\left(k_{1} \pi_{2}+k_{2} \pi_{1}\right)-4} V_{k_{1}+k_{2}, \pi_{1}+\pi_{2}}\left(\zeta_{2}\right)+ \\
& \mathcal{R E S}_{1}\left|\zeta_{1}-\zeta_{2}\right|^{2\left(k_{1} \pi_{2}+k_{2} \pi_{1}\right)-2} U_{k_{1}+k_{2}, \pi_{1}+\pi_{2}}\left(\zeta_{2}\right)+\ldots . .,
\end{aligned}
$$

where in general the lowest order vertex operator $V_{k, \pi}$ has appeared on the r.h.s. together with higher order ones. The corresponding residues are $\mathcal{R E} \mathcal{S}_{0}, \mathcal{R E S}_{1}$ and so on. To find this residues we have to compute the two-point correlation functions when $k_{1} \pi_{1}=k_{2} \pi_{2}=$ 0 . In the following we shall not discuss the contribution of the higher order operators on the r.h.s., but should mention that their contribution should also vanish. Thus, the only transitions which are permitted are between $U_{k, \pi}$ states of fixed helicity.

The two point correlation function of the vertex operator $U_{k, \pi}=\omega U_{X X}+2 \varphi U_{X \Pi}+\chi U_{\Pi \Pi}$ contains six cross terms which have the form [6]:

$$
\begin{gathered}
<U_{X X}^{\mu_{1} \nu_{1}} U_{X X}^{\mu_{2} \nu_{2}}>=\pi_{2}^{\mu_{1}} \pi_{2}^{\nu_{1}} \pi_{1}^{\mu_{2}} \pi_{1}^{\nu_{2}}\left|\zeta_{1}-\zeta_{2}\right|^{2\left(k_{1} \pi_{2}+k_{2} \pi_{1}\right)-4}, \\
<U_{X \Pi}^{\mu_{1} \nu_{1}} U_{X \Pi}^{\mu_{2} \nu_{2}}>=2\left\{\eta^{\mu_{1} \nu_{2}} \eta^{\nu_{1} \mu_{2}}-\eta^{\mu_{1} \nu_{2}} k_{2}^{\nu_{1}} \pi_{1}^{\mu_{2}}-\eta^{\nu_{1} \mu_{2}} \pi_{2}^{\mu_{1}} k_{1}^{\nu_{2}}+\right. \\
\left.+2 \pi_{2}^{\mu_{1}} k_{2}^{\nu_{1}} \pi_{1}^{\mu_{2}} k_{1}^{\nu_{2}}\right\}\left|\zeta_{1}-\zeta_{2}\right|^{2\left(k_{1} \pi_{2}+k_{2} \pi_{1}\right)-4}, \\
<U_{\Pi \Pi}^{\mu_{1} \nu_{1}} U_{\Pi \Pi}^{\mu_{2} \nu_{2}}>=k_{2}^{\mu_{1}} k_{2}^{\nu_{1}} k_{1}^{\mu_{2}} k_{1}^{\nu_{2}}\left|\zeta_{1}-\zeta_{2}\right|^{2\left(k_{1} \pi_{2}+k_{2} \pi_{1}\right)-4}, \\
<U_{X X}^{\mu_{1} \nu_{1}} U_{X \Pi}^{\mu_{2} \nu_{2}}>=\left(2 \pi_{2}^{\mu_{1}} \pi_{2}^{\nu_{1}} \pi_{1}^{\mu_{2}} k_{1}^{\nu_{2}}-\eta^{\nu_{1} \nu_{2}} \pi_{2}^{\mu_{1}} \pi_{1}^{\mu_{2}}-\eta^{\mu_{1} \nu_{2}} \pi_{2}^{\nu_{1}} \pi_{1}^{\mu_{2}}\right)\left|\zeta_{1}-\zeta_{2}\right|^{2\left(k_{1} \pi_{2}+k_{2} \pi_{1}\right)-4}, \\
<U_{X X}^{\mu_{1} \nu_{1}} U_{\Pi \Pi}^{\mu_{2} \nu_{2}}>=\left(\eta^{\mu_{1} \mu_{2}} \eta^{\nu_{1} \nu_{2}}-\eta^{\mu_{1} \mu_{2}} \pi_{2}^{\nu_{1}} k_{1}^{\nu_{2}}-\eta^{\nu_{1} \nu_{2}} \pi_{2}^{\mu_{1}} k_{1}^{\mu_{2}}+\right. \\
\left.+\pi_{2}^{\mu_{1}} k_{1}^{\mu_{2}} \pi_{2}^{\nu_{1}} k_{1}^{\nu_{2}}\right)\left|\zeta_{1}-\zeta_{2}\right|^{2\left(k_{1} \pi_{2}+k_{2} \pi_{1}\right)-4},
\end{gathered}
$$


$<U_{X \Pi}^{\mu_{1} \nu_{1}} U_{\Pi \Pi}^{\mu_{2} \nu_{2}}>=\left(2 \pi_{2}^{\mu_{1}} k_{2}^{\nu_{1}} k_{1}^{\mu_{2}} k_{1}^{\nu_{2}}-\eta^{\mu_{1} \mu_{2}} k_{2}^{\nu_{1}} k_{1}^{\nu_{2}}-\eta^{\mu_{1} \nu_{2}} k_{2}^{\nu_{1}} k_{1}^{\mu_{2}}\right)\left|\zeta_{1}-\zeta_{2}\right|^{2\left(k_{1} \pi_{2}+k_{2} \pi_{1}\right)-4}$

and the full two point correlation function is:

$$
\begin{aligned}
& <U_{k_{1}, \pi_{1}}, U_{k_{2}, \pi_{2}}>= \\
& =<\omega_{1} U_{X X}+2 \varphi_{1} U_{X \Pi}+\chi_{1} U_{\Pi \Pi}, \omega_{2} U_{X X}+2 \varphi_{2} U_{X \Pi}+\chi_{2} U_{\Pi \Pi}> \\
& =\mathcal{R E S}_{0} \quad\left|\zeta_{1}-\zeta_{2}\right|^{2\left(k_{1} \pi_{2}+k_{2} \pi_{1}\right)-4} \text {. }
\end{aligned}
$$

Using the above pairs of correlation functions (15) we can get

$$
\begin{aligned}
& \mathcal{R} \mathcal{E} \mathcal{S}_{0}=\left(\pi_{2} \omega_{1} \pi_{2}\right)\left(\pi_{1} \omega_{2} \pi_{1}\right)+ \\
& 2 \operatorname{tr}\left(\varphi_{1} \varphi_{2}\right)-2\left(\varphi_{1} k_{2}\right)\left(\pi_{1} \varphi_{2}\right)-2\left(\pi_{2} \varphi_{1}\right)\left(\varphi_{2} k_{1}\right)+4\left(\pi_{2} \varphi_{1} k_{2}\right)\left(\pi_{1} \varphi_{2} k_{1}\right)+ \\
& \left(k_{2} \chi_{1} k_{2}\right)\left(k_{1} \chi_{2} k_{1}\right)+ \\
& 2\left(\pi_{2} \omega_{1} \pi_{2}\right)\left(\pi_{1} \varphi_{2} k_{1}\right)-\left(\pi_{2} \omega_{1}\right)\left(\pi_{1} \varphi_{2}\right)-\left(\omega_{1} \pi_{2}\right)\left(\pi_{1} \varphi_{2}\right)+ \\
& \operatorname{tr}\left(\omega_{1} \chi_{2}\right)-\left(\omega_{1} \pi_{2}\right)\left(\chi_{2} k_{1}\right)-\left(\pi_{2} \omega_{1}\right)\left(k_{1} \chi_{2}\right)+\left(\pi_{2} \omega_{1} \pi_{2}\right)\left(k_{1} \chi_{2} k_{1}\right)+ \\
& \left.2\left(\pi_{1} \omega_{2} \pi_{1}\right)\left(\pi_{2} \varphi_{1} k_{2}\right)\right)-\left(\pi_{1} \omega_{2}\right)\left(\pi_{2} \varphi_{1}\right)-\left(\omega_{2} \pi_{1}\right)\left(\pi_{2} \varphi_{1}\right)+ \\
& 2\left(\pi_{2} \varphi_{1} k_{2}\right)\left(k_{1} \chi_{2} k_{1}\right)-\left(\varphi_{1} k_{2}\right)\left(\chi_{2} k_{1}\right)-\left(\varphi_{1} k_{2}\right)\left(k_{1} \chi_{2}\right)+ \\
& \operatorname{tr}\left(\chi_{1} \omega_{2}\right)-\left(\chi_{1} k_{2}\right)\left(\omega_{2} \pi_{1}\right)-\left(k_{2} \chi_{1}\right)\left(\pi_{1} \omega_{2}\right)+\left(k_{2} \chi_{1} k_{2}\right)\left(\pi_{1} \omega_{2} \pi_{1}\right)+ \\
& 2\left(k_{2} \chi_{1} k_{2}\right)\left(\pi_{1} \varphi_{2} k_{1}\right)-\left(\chi_{1} k_{2}\right)\left(\varphi_{2} k_{1}\right)-\left(k_{2} \chi_{1}\right)\left(\varphi_{2} k_{1}\right)
\end{aligned}
$$

The vertex operator $V_{k_{1}+k_{2}, \pi_{1}+\pi_{2}}$ should have anomalous dimension $\Delta$ equal to two (5) $2\left(k_{1}+k_{2}\right) \cdot\left(\pi_{1}+\pi_{2}\right)=2\left(k_{1} \pi_{2}+k_{2} \pi_{1}\right)=2$, therefore the transition amplitude to the state $V_{k, \pi}(0) \mid 0>$ is equal to zero if

$$
\mathcal{R E S}_{0}=0, \quad \text { at } \quad k_{1} \pi_{2}+k_{2} \pi_{1}=1 .
$$

In summary we have the system of equations which define the tensors $\omega, \varphi$ and $\chi$ : they are the equations (11),(12) and (13) together with (17) and (16).

When the above zero residue condition (17) is fulfilled then the three point space-time vertex function will be given by the residue $\mathcal{R} \mathcal{E} \mathcal{S}_{1}$ :

$$
U_{k_{1}, \pi_{1}}\left(\zeta_{1}\right) U_{k_{2}, \pi_{2}}\left(\zeta_{2}\right) \simeq \mathcal{R} \mathcal{E} \mathcal{S}_{1}\left|\zeta_{1}-\zeta_{2}\right|^{2\left(k_{1} \pi_{2}+k_{2} \pi_{1}\right)-2} \quad U_{k_{1}+k_{2}, \pi_{1}+\pi_{2}}\left(\zeta_{2}\right)+\ldots .
$$

at $\Delta=2\left(k_{1}+k_{2}\right) \cdot\left(\pi_{1}+\pi_{2}\right)+2=2\left(k_{1} \pi_{2}+k_{2} \pi_{1}\right)+2=2$, that is

$$
k_{1} \pi_{2}+k_{2} \pi_{1}=0 \text {. }
$$

The general solution of the equations (11),(12), (13) and (17) has not been found yet, but some particular two-parameter family of solutions of fixed helicity operators $U_{k, \pi}^{T}$ can be found. The solution will be presented in the next section. Unfortunately the scattering

of this family of states creates the state which does not belong to the same family and one should find more general solution. Nevertheless this exercise can provide necessary information about the general solution.

\section{Examples of Fixed Helicity Vertex Operators}

Let us consider the derivatives of the operator $V_{k, \pi}$

$$
U_{k, \pi}^{L}=\partial_{\zeta} \partial_{\bar{\zeta}} V_{k, \pi}=:\left(i k \cdot \partial_{\zeta} X+i \pi \cdot \partial_{\zeta} \Pi\right)\left(i k \cdot \partial_{\bar{\zeta}} X+i \pi \cdot \partial_{\bar{\zeta}} \Pi\right) e^{i k \cdot X+i \pi \cdot \Pi}:
$$


If one takes the invariant $(k \cdot \pi)=0$, then the $U_{k, \pi}^{L}$ is a primary field of anomalous dimension two and is of the same type as $U_{k, \pi}$ in (7). Comparing it with the general form of the vertex operator $U_{k, \pi}$ in (8), one can conclude that it is the solution of (12) and (13) of the form

$$
\omega_{\mu \nu}=a k_{\mu} k_{\nu}, \quad \varphi_{\mu \nu}=a k_{\mu} \pi_{\nu}, \quad \chi_{\mu \nu}=a \pi_{\mu} \pi_{\nu}
$$

This state corresponds to the longitudinal mode and should decouple from the physical amplitudes. Indeed, suppose that we are scattering two longitudinal modes which are given by the above tensors. Then using our two-point correlation function and the expression for the corresponding residue (16) one can find

$$
\begin{aligned}
U_{k_{1}, \pi_{1}}^{L}\left(\zeta_{1}\right) U_{k_{2}, \pi_{2}}^{L}\left(\zeta_{2}\right) \simeq\left(k_{1} \pi_{2}+k_{2} \pi_{1}\right)^{2}\left(k_{1} \pi_{2}+k_{2} \pi_{1}-1\right)^{2} & \\
& \left|\zeta_{1}-\zeta_{2}\right|^{2\left(k_{1} \pi_{2}+k_{2} \pi_{1}\right)-4} V_{k_{1}+k_{2}, \pi_{1}+\pi_{2}}\left(\zeta_{2}\right)+\ldots .
\end{aligned}
$$

Because $V_{k, \pi}$ should have anomalous dimension equal to two the pole on the r.h.s. is at the point $k_{1} \pi_{2}+k_{2} \pi_{1}=1(17)$. This implies that the residue of the corresponding pole is equal to zero

$$
\mathcal{R} \mathcal{E} \mathcal{S}_{0}=\left(k_{1} \pi_{2}+k_{2} \pi_{1}\right)^{2}\left(k_{1} \pi_{2}+k_{2} \pi_{1}-1\right)^{2}=0 .
$$

Our aim now is to find new solution of the equations (12), (13) with the same property, that is in their operator product the corresponding residue to the $V_{k, \pi} \mid 0>$ state is equal to zero (17). The condition for that is that the polynomial (16) should vanish at the pole (17): $k_{1} \pi_{2}+k_{2} \pi_{1}=\alpha+\beta=1$. The scalars $k_{1} \pi_{2}$ and $k_{2} \pi_{1}$ will appear frequently in our calculations and we shall use special notation for them: $\alpha=k_{1} \pi_{2}$ and $\beta=k_{2} \pi_{1}$.

Let us consider the three-parameter solution of equations (12), (13) for $U_{k, \pi}$ of the form:

$$
\omega_{\mu \nu}=(1+\varepsilon) k_{\mu} k_{\nu}, \quad \varphi_{\mu \nu}=(1+\delta) k_{\mu} \pi_{\nu}, \quad \chi_{\mu \nu}=(1+\varrho) \pi_{\mu} \pi_{\nu}
$$

where $\varepsilon, \delta$ and $\varrho$ are unknown. Substituting this parameterization into (16) we shall get

$$
\begin{aligned}
\mathcal{R E S}_{0} & =\left((1+\varepsilon)^{2}+(1+\varrho)^{2}\right) \alpha^{2} \beta^{2} \\
& +(1+\delta)^{2} 2 \alpha \beta(1-\alpha-\beta+2 \alpha \beta) \\
& +(1+\varepsilon)(1+\delta) 2 \alpha \beta\left(\alpha^{2}+\beta^{2}-\alpha-\beta\right) \\
& +(1+\varepsilon)(1+\varrho)\left(\alpha^{2}+\beta^{2}-2 \alpha^{3}-2 \beta^{3}+\alpha^{4}+\beta^{4}\right) \\
& +(1+\delta)(1+\varrho) 2 \alpha \beta\left(\alpha^{2}+\beta^{2}-\alpha-\beta\right) .
\end{aligned}
$$

At the pole $\alpha+\beta=1$ the corresponding residue should vanish, thus substituting $\alpha+\beta=1$ into the last equation we shall get

$$
\begin{aligned}
\mathcal{R E S}_{0} & =\left[(1+\varepsilon)^{2}+(1+\varrho)^{2}+4(1+\delta)^{2}\right. \\
& -4(1+\varepsilon)(1+\delta) \\
& +2(1+\varepsilon)(1+\varrho) \\
& -4(1+\delta)(1+\varrho)] \quad \alpha^{2}\left(1-\alpha^{2}\right) \\
& =[(1+\varepsilon)+(1+\varrho)-2(1+\delta)]^{2} \quad \alpha^{2}\left(1-\alpha^{2}\right) .
\end{aligned}
$$

The residue $\mathcal{R} \mathcal{E} \mathcal{S}_{0}$ is equal to zero if

$$
\varepsilon+\varrho-2 \delta=0 .
$$


The last equation gives us two-parameter family of operators with required property

$$
\begin{array}{r}
U_{k, \pi}^{T}(\zeta)=(1+\varepsilon):\left(k \cdot \partial_{\zeta} X k \cdot \partial_{\bar{\zeta}} X-\pi \cdot \partial_{\zeta} \Pi \pi \cdot \partial_{\bar{\zeta}} \Pi\right) e^{i k \cdot X(\zeta)+i \pi \cdot \Pi(\zeta)}: \\
(1+\delta):\left(k \cdot \partial_{\zeta} X \pi \cdot \partial_{\bar{\zeta}} \Pi+k \cdot \partial_{\bar{\zeta}} X \pi \cdot \partial_{\zeta} \Pi+2 \pi \cdot \partial_{\zeta} \Pi \pi \cdot \partial_{\bar{\zeta}} \Pi\right) e^{i k \cdot X(\zeta)+i \pi \cdot \Pi(\zeta)}:
\end{array}
$$

Thus we can select two independent vertex operators

$$
U_{k, \pi}^{T_{1}}(\zeta)=:\left(k \cdot \partial_{\zeta} X k \cdot \partial_{\bar{\zeta}} X-\pi \cdot \partial_{\zeta} \Pi \pi \cdot \partial_{\bar{\zeta}} \Pi\right) W_{k, \pi}(\zeta):
$$

and

$$
U_{k, \pi}^{T_{2}}(\zeta)=:(k \cdot \partial X \pi \cdot \bar{\partial} \Pi+k \cdot \bar{\partial} X \pi \cdot \partial \Pi+2 \pi \cdot \partial \Pi \pi \cdot \bar{\partial} \Pi) W_{k, \pi}(\zeta):,
$$

where for both of them $(k \cdot \pi)=0$ and $W_{k, \pi}(\zeta)=\exp [i k \cdot X(\zeta)+i \pi \cdot \Pi(\zeta)]$.

We have to compute now the next term in the OPE of these operators as in (14) in order to see if there are nonzero transitions to the states not belonging to this family. We have

$$
\begin{gathered}
U_{k_{1}, \pi_{1}}^{T_{1}}\left(\zeta_{1}\right) U_{k_{2}, \pi_{2}}^{T_{1}}\left(\zeta_{2}\right) \simeq \\
\left(\alpha^{2}-\beta^{2}\right):\left(k_{1} \cdot \partial_{\zeta} X k_{1} \cdot \partial_{\bar{\zeta}} X-\pi_{1} \cdot \partial_{\zeta} \Pi \pi_{1} \cdot \partial_{\bar{\zeta}} \Pi-k_{2} \cdot \partial_{\zeta} X k_{2} \cdot \partial_{\bar{\zeta}} X+\pi_{2} \cdot \partial_{\zeta} \Pi \pi_{2} \cdot \partial_{\bar{\zeta}} \Pi\right) \\
+\alpha \beta \quad\left(k_{1} \cdot \partial_{\zeta} X k_{2} \cdot \partial_{\bar{\zeta}} X+k_{2} \cdot \partial_{\zeta} X k_{1} \cdot \partial_{\bar{\zeta}} X+\pi_{1} \cdot \partial_{\zeta} \Pi \pi_{2} \cdot \partial_{\bar{\zeta}} \Pi+\pi_{2} \cdot \partial_{\zeta} \Pi \pi_{1} \cdot \partial_{\bar{\zeta}} \Pi\right) \\
\left|\zeta_{1}-\zeta_{2}\right|^{2\left(k_{1} \pi_{2}+k_{2} \pi_{1}\right)-2} W_{k_{1}+k_{2}, \pi_{1}+\pi_{2}}\left(\zeta_{2}\right):+\ldots . .,
\end{gathered}
$$

and at the pole $\alpha+\beta=0$ we get

$$
\begin{gathered}
U_{k_{1}, \pi_{1}}^{T_{1}}\left(\zeta_{1}\right) U_{k_{2}, \pi_{2}}^{T_{1}}\left(\zeta_{2}\right) \simeq \\
\alpha \beta:\left(k_{1} \cdot \partial_{\zeta} X k_{2} \cdot \partial_{\bar{\zeta}} X+k_{2} \cdot \partial_{\zeta} X k_{1} \cdot \partial_{\bar{\zeta}} X+\pi_{1} \cdot \partial_{\zeta} \Pi \pi_{2} \cdot \partial_{\bar{\zeta}} \Pi+\pi_{2} \cdot \partial_{\zeta} \Pi \pi_{1} \cdot \partial_{\bar{\zeta}} \Pi\right) \\
\left|\zeta_{1}-\zeta_{2}\right|^{2\left(k_{1} \pi_{2}+k_{2} \pi_{1}\right)-2} W_{k_{1}+k_{2}, \pi_{1}+\pi_{2}}\left(\zeta_{2}\right):+\ldots \ldots .
\end{gathered}
$$

The r.h.s. can not be collected into the $U_{k_{1}+k_{2}, \pi_{1}+\pi_{2}}^{T_{1}}$ operator. Therefore the fusion of two $U^{T_{1}}$ 's of the above form does not scatter to the same state.

The same happens with the second operator $U^{T_{2}}$, indeed

$$
\begin{gathered}
U_{k_{1}, \pi_{1}}^{T_{2}}\left(\zeta_{1}\right) U_{k_{2}, \pi_{2}}^{T_{2}}\left(\zeta_{2}\right) \simeq \\
\alpha \beta \quad:\left(k_{1} \cdot \partial_{\zeta} X k_{2} \cdot \partial_{\bar{\zeta}} X+k_{2} \cdot \partial_{\zeta} X k_{1} \cdot \partial_{\bar{\zeta}} X+\pi_{1} \cdot \partial_{\zeta} \Pi \pi_{2} \cdot \partial_{\bar{\zeta}} \Pi+\pi_{2} \cdot \partial_{\zeta} \Pi \pi_{1} \cdot \partial_{\bar{\zeta}} \Pi+\right. \\
\left.k_{1} \cdot \partial_{\zeta} X \pi_{2} \cdot \partial_{\bar{\zeta}} \Pi+\pi_{2} \cdot \partial_{\zeta} \Pi k_{1} \cdot \partial_{\bar{\zeta}} X+\pi_{1} \cdot \partial_{\zeta} \Pi k_{2} \cdot \partial_{\bar{\zeta}} X+k_{2} \cdot \partial_{\zeta} X \pi_{1} \cdot \partial_{\bar{\zeta}} \Pi\right) \\
\left|\zeta_{1}-\zeta_{2}\right|^{2\left(k_{1} \pi_{2}+k_{2} \pi_{1}\right)-2} W_{k_{1}+k_{2}, \pi_{1}+\pi_{2}}\left(\zeta_{2}\right):+\ldots . .(3)
\end{gathered}
$$

\section{Conclusion}

The vertex operators of the tensionless string model have been constructed in [6] as polynomials of the field derivatives $\partial_{\zeta}^{m_{1}} X^{\mu_{1}}, \ldots, \partial_{\bar{\zeta}}^{\tilde{m}_{j}} \Pi^{\tilde{\mu}_{j}} e^{i k \cdot X(\zeta)+i \pi \cdot \Pi(\zeta)}$ and have anomalous dimension $\Delta=2(k \cdot \pi)+m_{1}+\tilde{m}_{1}+\ldots . .+m_{j}+\tilde{m}_{j}$. Of special importance is the vertex operator for fixed helicity states

$$
U_{k, \pi}=\omega_{\mu \nu} U_{X X}^{\mu \nu}+2 \varphi_{\mu \nu} U_{X \Pi}^{\mu \nu}+\chi_{\mu \nu} U_{\Pi \Pi}^{\mu \nu},
$$


which is quadratic in field derivatives. Its anomalous dimension is $\Delta=2(k \cdot \pi)+2=2$, that is $(k \cdot \pi)=0$. The last invariant coincides with the length of the second Casimir operator of the Poincaré group $W=(k \cdot \pi)^{2}=0$. Therefore it creates fixed helicity states. All other operators have $W \neq 0$ and create continuous spin representations - CSR, which are longitudinal modes in accordance with the conjecture made in [2].

In order to justify this picture one should prove that all transition amplitudes to the states of continuous spin representations are equal to zero and that the only nonzero amplitudes are between fixed helicity states created by the $U_{k, \pi}$ operator. We don't know the full solution of this problem, but have been able to derive the conditions (12), (13) together with (17) and (16) on the operator $U_{k, \pi}$ which guarantee the zero transition amplitude to the state created by the lowest order CSR operator $V_{k, \pi}$ in tree approximation.

If the calculation of the conformal dimensions (4) for the vertex operators (1) was for some reason incorrect, then the conclusion about $V$ and $U$ operators can in principle be changed. For example, if there is some undefined source of contribution to the conformal dimension in (4), then the conclusion indeed can change. Suppose that there is an additional term to the conformal dimension like in the following formula $\Delta=2(k \cdot \pi)+m_{1}+\tilde{m}_{1}+\ldots . .+m_{j}+\tilde{m}_{j}+2$, then $V$ becomes physical operator and $U$ unphysical because of additional 2. The Abelian constraint $\Theta$ can be such a source of additional contribution, through the corresponding Faddeev-Papov ghosts. But the Faddeev-Popov determinant is trivial here because there are no derivatives in the Abelian constraint $\Theta=\Pi^{2}-1=0$.

Let me also mention that it is generally expected that the tensionless limit $\alpha^{\prime} \rightarrow \infty$ of the standard string theory with the Nambu-Goto area action should have massless spectrum, because all masses at every level tend to zero as $M_{N}^{2}=(N-1) / \alpha^{\prime} \rightarrow 0[5]$. Of course this simple conclusion ignores the importance of the high genus $g$ diagrams, the contribution of which $A_{g}$ is exponentially large compared to the tree level diagram [5]. The ratio of the corresponding scattering amplitudes behaves as $A_{g+1} / A_{g} \simeq \exp \left\{\alpha^{\prime} s / g^{2}\right\}$ and makes any perturbative statement unreliable and requires therefore nonperturbative treatment of the problem ${ }^{4}$.

The tensionless model with perimeter action suggested in $[1,2,3]$ does not appear as a $\alpha^{\prime} \rightarrow \infty$ limit of the standard string theory, as one could probably think, but has a tensionless character by its geometrical nature [3]. Therefore it remains mainly unclear at the moment how these two models are connected. However the perimeter model shares many properties with the area strings in the sense that it has world-sheet conformal invariance, contains the corresponding Virasoro algebra, which is extended by additional Abelian generators. This makes mathematics used in the perimeter model very close to the standard string theory and allows to compute its spectrum [1,2] and to construct the appropriate vertex operators [6].

Comparing literally the spectrum of these two models one can see that instead of exponential growing of states in the standard string theory, in the perimeter case we have only linear growing of physical states. In this respect the number of states in the perimeter model is much less compared with the standard string theory and is larger compared with the standard field theory models of the Yang-Mills type. From this point of view it is therefore much closes to the quantum field theory rather than to the standard string theory. At the same time its formulation and the symmetry structure is more

\footnotetext{
${ }^{4}$ The different aspects and models of tensionless theories can be found in $[9,10,11,12,13,14,15]$.
} 
string-theoretical. Perhaps there should be strong nonperturbative rearrangement of the spectrum in the limit $\alpha^{\prime} \rightarrow \infty$ before the spectrum of the area and the perimeter strings can become close to each other.

I would like to thank Luis Alvarez-Gaume, Ignatios Antoniadis, Lars Brink and Kumar Narain for stimulating discussions and CERN Theory Division for hospitality.

\section{Appendix}

The analysis of the physical Fock space is based on the equations which follow from Abelian constraint $\Theta=\Pi^{2}-1=0[2]$. The $\Pi^{\mu}=\frac{1}{2}\left(\Pi_{L}^{\mu}\left(\zeta^{+}\right)+\Pi_{R}^{\mu}\left(\zeta^{-}\right)\right)$field is

$$
\begin{aligned}
& \Pi_{L}^{\mu}=e^{\mu}+k^{\mu} \zeta^{+}+i \sum_{n \neq 0} \frac{1}{n} \alpha_{n}^{\mu} e^{-i n \zeta^{+}}, \\
& \Pi_{R}^{\mu}=e^{\mu}+k^{\mu} \zeta^{-}+i \sum_{n \neq 0} \frac{1}{n} \tilde{\alpha}_{n}^{\mu} e^{-i n \zeta^{-}} .
\end{aligned}
$$

and for the lowest excitations it has the form

$$
\Pi^{\mu}(\sigma, \tau)=e^{\mu}+k^{\mu} \tau+\frac{i}{2} \alpha_{1}^{\mu} e^{-i \zeta^{+}}-\frac{i}{2} \alpha_{-1}^{\mu} e^{i \zeta^{+}}+\ldots
$$

Thus the constraint equation $\Pi^{2}-1=0$ will take the form

$$
\begin{aligned}
& \Pi^{2}-1=e^{2}-1+\frac{1}{2} \alpha_{1} \alpha_{-1}+ \\
& k^{2} \tau^{2}+2(e \cdot k) \tau+ \\
& i\left(k \cdot \alpha_{1}\right) \tau e^{-i \zeta^{+}} \quad-i\left(k \cdot \alpha_{-1}\right) \tau e^{i \zeta^{+}}+ \\
& i\left(e \cdot \alpha_{1}\right) e^{-i \zeta^{+}}-i\left(e \cdot \alpha_{-1}\right) e^{i \zeta^{+}}- \\
& -\frac{1}{4} \alpha_{1} \alpha_{1} e^{-2 i \zeta^{+}}-\frac{1}{4} \alpha_{-1} \alpha_{-1} e^{2 i \zeta^{+}} \ldots . .=0 .
\end{aligned}
$$

In components it is equivalent to the system of constraint equations (formulas (32) in [2])

$$
\begin{gathered}
k^{2}=0, \quad k \cdot e=0, \quad e^{2}=1-\frac{1}{2} \alpha_{1} \alpha_{-1}, \\
\left(k \cdot \alpha_{ \pm 1}\right)=0, \quad\left(e \cdot \alpha_{ \pm 1}\right)=0 . \\
\alpha_{1} \alpha_{1}=0, \quad \alpha_{-1} \alpha_{-1}=0 .
\end{gathered}
$$

The question is what they mean for the oscillators $\alpha_{ \pm 1}^{\mu}$.

To find an answer let us consider the world-sheet time $\tau$ evolution of the field $\Pi(\sigma, \tau)$. We have to fix the initial conditions of the field $\Pi(\sigma, \tau)$. For that we have to define the polarization vector $e$, the momentum $\mathrm{k}$ and the coefficients $\alpha_{ \pm n}$ in (32). Because of the existence of the constraints, the variables e, $\mathrm{k}, \alpha_{ \pm n}$ are not dynamically independent. The equations (36) tell us that they are transverse and longitudinal at the same time. The equations (37) tell us that they are light-like.

The polarization vector $e$ can be equal to $e_{1}$ or $e_{2}$ where $e_{1}$ and $e_{2}$ are two fixed perpendicular $e_{1} \cdot e_{2}=0$ purely spatial vectors $-e_{0}^{2}+\vec{e}^{2}=1$ (i.e., whose $e_{0}$ component is 0 
and for simplicity we are considering four-dimensional space-time) which are orthogonal to the fixed light-like momentum vector $\mathrm{k}$

$$
k \cdot e_{1}=k \cdot e_{2}=0
$$

If we take the initial condition as $\left(k, e_{1}\right)$ then the constraint equations tell us that the physical oscillations of $\alpha_{1}$ are those which are perpendicular to $\mathrm{k}$ and to $e_{1}$

$$
\left(k \cdot \alpha_{ \pm 1}\right)=0, \quad\left(e_{1} \cdot \alpha_{ \pm 1}\right)=0,
$$

thus

$$
\alpha_{ \pm 1}^{\mu}=\alpha_{ \pm 1} k^{\mu}+B_{ \pm} e_{2}^{\mu}
$$

and substituting it into the last equation (14) we shall get $\alpha_{1} \alpha_{1}=B_{+}^{2}=0$, that is $B_{+}=0$. We have also $\alpha_{-1} \alpha_{-1}=B_{-}^{2}=0$, thus $B_{-}=0$.

If we take the initial conditions in the second possible form $\left(k, e_{2}\right)$, then the constraint equations will tell us that physical oscillations of the oscillators $\alpha_{ \pm 1}$ are in the $e_{1}$ direction and the oscillations in the $e_{2}$ direction are forbidden

$$
\left(k \cdot \alpha_{ \pm 1}\right)=0, \quad\left(e_{2} \cdot \alpha_{ \pm 1}\right)=0
$$

thus

$$
\alpha_{ \pm 1}^{\mu}=\alpha_{ \pm 1} k^{\mu}+B_{ \pm} e_{1}^{\mu}
$$

and from the last equation (14) we shall get $B_{ \pm}=0$. Therefore in all cases the oscillators $\alpha_{ \pm 1}^{\mu}$ are purely longitudinal

$$
\alpha_{ \pm 1}^{\mu}=\alpha_{ \pm 1} k^{\mu}
$$

and it is natural to introduce the scalar oscillators $\alpha_{ \pm 1}$. The $\Pi$ field will take the form

$$
\Pi^{\mu}(\sigma, \tau)=e^{\mu}+k^{\mu}\left\{\tau+\frac{i}{2} \alpha_{1} e^{-i \zeta^{+}}-\frac{i}{2} \alpha_{-1} e^{i \zeta^{+}}\right\}+\ldots
$$

In general case we shall have

$$
\Pi^{\mu}(\sigma, \tau)=e^{\mu}+k^{\mu}\left\{\tau+i \sum_{n \neq 0} \frac{1}{2 n}\left(\alpha_{n} e^{-i n \zeta^{+}}+\tilde{\alpha}_{n}^{\mu} e^{-i n \zeta^{-}}\right)\right\} .
$$

The last formula has clear physical interpretation: it defines the polarization vector $e^{\mu}$ up to a large gauge transformation of the from

$$
e^{\mu} \rightarrow e^{\mu}+k^{\mu} f\left(\tau, \sigma, a_{n}\right) .
$$

This was the classical consideration. In the course of covariant quantization our constraints will be translated into the constraints imposed on the wave function [2]. Because the oscillators $\alpha_{ \pm n}^{\mu}=k^{\mu} \alpha_{ \pm n}$ are pure longitudinal, they create only zero norm states. 


\section{References}

[1] G. K. Savvidy, Phys.Lett. B 552 (2003) 72.

[2] G. K. Savvidy, "Tensionless strings: Physical Fock space and higher spin fields," Int. J. Mod. Phys. A 19, (2004) 3171-3194; hep-th/0310085.

[3] G. Savvidy and K. Savvidy. Mod.Phys.Lett. A8 (1993) 2963; Int.J.Mod.Phys. A8 (1993) 3993; R.V.Ambartzumian and et al, Phys.Lett. B275 (1992) 99; G. Savvidy. JHEP 0009 (2000) 044; R. Manvelian and G. Savvidy,Phys.Lett.B533 (2002) 138

[4] I. Antoniadis and G. Savvidy, "Physical Fock space of tensionless strings," arXiv:hepth/0402077.

[5] D.Gross, Phys.Rev.Lett. 60 (1988) 1229

[6] L. Alvarez-Gaume, I. Antoniadis, L. Brink, K. Narain and G. Savvidy "Tensionless Strings, Vertex Operators and Scattering Amplitudes ", Preprint CERN-PHTH/2004-095 and NRCPS-HE-2004-13, to be published

[7] E. Wigner, in Theoretical Physics ed. A.Salam (International Atomic Energy, Vienna, 1963) p 59; E. Wigner, Ann. Math. 40 (1939) 149.

[8] L. Brink, A. M. Khan, P. Ramond and X. Xiong, J. Math. Phys. 43 (2002) 6279

[9] H. J. de Vega and N. Sanchez, Phys. Lett. B 197 (1987) 320.

[10] D. Amati, M. Ciafaloni and G. Veneziano, Phys. Lett. B 216 (1989) 41.

[11] M. Gasperini, N. Sanchez and G. Veneziano, Nucl. Phys. B 364 (1991) 365.

[12] U. Lindstrom, B. Sundborg and G. Theodoridis, Phys. Lett. B 253 (1991) 319.

[13] H. J. De Vega and A. Nicolaidis, Phys. Lett. B 295 (1992) 214.

[14] F. Lizzi, Mod. Phys. Lett. A 9, 1495 (1994)

[15] I. Bakas and C. Sourdis, "On the tensionless limit of gauged WZW models," arXiv:hep-th/0403165.

[16] M. Fierz and W. Pauli, Proc.Roy.Soc. A173 (1939) 211

W.Rarita and J.Schwinger, Phys.Rev. 60 (1941) 61

J.Schwinger, Particles, Sourses, and Fields (Addison-Wesley, Reading, MA, 1970)

[17] L. P. S. Singh and C. R. Hagen, Phys.Rev. D9 (1974) 898, 910

[18] C.Fronsdal, Massless fields with integer spin, Phys.Rev. D18 (1978) 3624

[19] A. K. Bengtsson, I. Bengtsson and L. Brink, Nucl. Phys. B 227 (1983) 41;Nucl. Phys. B 227 (1983) 31. 